psychopraxis. neuropraxis $2020 \cdot 23: 156-157$ https://doi.org/10.1007/s00739-020-00664-2

(c) Springer-Verlag GmbH Austria, ein Teil von Springer Nature 2020
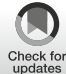

\title{
Walter Struhal
}

Abteilung für Neurologie, Universitätsklinikum Tulln, Karl Landsteiner Privatuniversität für Gesundheitswissenschaften, Tulln, Österreich

\section{Relevante Themen in der Neurologie und Psychiatrie}

Geschätzte Leserinnen, geschätzte Leser!

Welche Themen in der Neurologie und in der Psychiatrie sind derzeit von besonders hoher Relevanz für das Management unserer Patienten?

In der derzeitigen Situation der alles überschattenden SARS-CoV2-Thematik könnte man meinen, COVID-19 wäre die Antwort.

\section{Neurologie und Psychiatrie im Kontext von COVID-19}

Tatsächlich zeigen sich zunehmend auch neurologische und psychiatrische Komplikationen dieser Erkrankung, wie wir sie auch schon von den Erfahrungen mit SARS antizipieren konnten. Vor allem cerebrovaskuläre Komplikationen sind in einer aktuellen Studie von Varatharaj et al. häufig ( $n=77,62 \%$ der auswertbaren 125 Patienten) [1]; davon erlitten $74 \%$ einen ischämischen Schlaganfall, 9\% eine intrakranielle Blutung, ein Patient eine ZNS-Vaskulitis. Darüber hinaus waren neuropsychiatrische bzw. psychiatrische Syndrome häufig mit $31 \%$ ( $n=39$ ): $23 \%$ unspezifische Enzephalopathie, $18 \%$ Enzephalitis und ganze 59\% $(n=23)$ psychiatrische Erkrankungen, von denen $92 \%$ neu aufgetreten waren: $43 \%$ neu aufgetretene Psychose, 26\% neurokognitive demenzartige Syndrome und $17 \%$ affektive Störungen.

Ohne Frage sind wir gefordert, sehr aufmerksam neurologische und psychiatrische Symptome und Syndrome bei SARS-CoV2 in einen pathophysiologischen Kontext zu bringen. Wir sind bzgl. SARS-CoV2 recht früh in der Erklimmung unserer noch steilen Lernkurve, speziell was unsere Fächer betrifft. Unseren Kolleginnen und Kollegen weltweit geht es nicht anders. Einzigartig ist aber die globale und global parallele Relevanz eines komplett neuen Gesundheitsthemas und damit folgt mein Aufruf, weltweit Daten zu nutzen, um die Lernkurve neurologischer und psychiatrischer Syndrome in Verbindung mit SARS-CoV2 nicht in einzelnen Institutionen, einzelnen Ländern, sondern weltweit zu bezwingen. Eine Möglichkeit dazu stellt Open Science dar. Dies ist eine Alternative des 21. Jahrhunderts zur Publikation eines Papers und ist ebenso zitierbar. Ziel ist es nicht, Papers zu publizieren (oder nicht nur), sondern vor allem Daten. Ein schönes Beispiel auf nationaler Ebene stellt oben angeführte Studie dar. Derzeit sind unsere Felder in diesem innovativen Publikationsmodell noch recht spärlich vertreten. Bestmögliche Chancen, die Lernkurve unserer Fächer mit dem neuen Thema COVID-19 zu bezwingen, sind, dieses Publikationsmodell für jeden Einzelfall, den wir sehen, zu nutzen und so der globalen Forschungsgemeinde eine Chance zu geben, viele Fälle $\mathrm{zu}$ aggregieren und damit rascher ein weltweites Management zu entwickeln.

Allerdings ist jedenfalls Vorsicht geboten, eine Koinfektion mit SARS-CoV2 ohne pathophysiologische Erklärung zur Kausalität einer neurologischen oder psychiatrischen Erkrankung zu erklären. Ganz im Gegenteil haben sich das Spektrum und die Anforderungen an die Neurologie und Psychiatrie nicht verändert bzw. durch die Pandemie sogar erweitert. Zusätzlich adaptieren wir unsere Arbeit durch mannigfaltige Organisationsnotwendigkeiten der Hygiene. Dennoch hat vor allem die Versorgung unserer Patienten trotz einer weltweiten und einzigartigen Epidemie nach unseren hohen Standards zu erfolgen und gerade jetzt noch mehr sind wir verpflichtet, als Advokat der Interessen neurologischer und psychiatrischer $\mathrm{Pa}$ tienten $\mathrm{zu}$ agieren [2] und keinesfalls eine implizite Reduktion der Versorgung neurologischer oder psychiatrischer $\mathrm{Pa}$ tienten zuzulassen. Die Frage COVID-19 oder eine andere Erkrankung stellt sich ja in der täglichen Praxis unserer Fächer am Krankenbett nicht. Vielmehr sind unsere Erkrankungen weiterhin in der Dringlichkeit, der Schwere für unsere Patienten vorhanden und wenn eine Koinfektion mit SARS-Cov2 besteht, sind zusätzliche Maßnahmen zu ergreifen, die dem Schutz von Patientinnen und Patienten, dem Schutz von Mitarbeiterinnen und Mitarbeitern Sorge tragen.

\section{Aktuelle Themen}

In der vorliegenden Ausgabe haben wir uns den klinischen Herausforderungen gestellt, die gerade in der Patientenversorgung eine entscheidende Rolle spielen:

\section{Ihnen ist sicher das Konzept der behandelbaren Demenzen vertraut - bedeutet das also, es gibt unbehandelbare Demenzen?}

Umkehrschlüsse sind einerseits menschlich, andererseits äußerst gefährlich. Univ.-Prof. DDr. P. Fischer diskutiert anhand dreier Fälle die Wichtigkeit, gegen diesen Trugschluss einzutreten.

\section{Was können wir beim Schlaganfall unter NOAK (nicht- Vitamin-K-abhängigen oralen Antikoagulanzien) tun? Und wie behandeln wir multidisziplinär Dysphagie nach Schlaganfall?}

Diese hochaktuellen Themen werden von OÄ Dr. C. Brunner et al., Dr. S. Beirer und Logopädin PhDr M. Trapl-Grundschober, MAS, MSc. einprägsam begangen. 


\section{Wenn Epilepsiepatienten Verhaltensauffälligkeiten entwickeln - ist es das Antikonvulsivum?}

Dr. T. Kapfer und Dr. E. Kacar et al. haben die aktuellen Daten aufgearbeitet und geben klinische Hinweise aus diesem Dilemma.

\section{Das Konzept der Mentalisierung bei Störungen aus dem schizophrenen Formenkreis - wie treten Defizite der Mentalisierung in Erscheinung?}

Als Neurologe fand ich besonders das Konzept der Mentalisierung und deren spezifische Ausformungen sehr interessant. Diese Lektüre gibt auch der Neurologie wertvolle Stimuli für den Patientenkontakt.

Dr. P. Grafenauer stellt besondere Aspekte bei der Betreuung von Myasthenia-gravis-Patienten vor und geht dabei auf ganz wichtige und klinisch relevante Aspekte der Patientenversorgung ein.
Ich hoffe, Sie finden die Lektüre ebenso spannend wie hilfreich in unserer täglichen Aufgabe.

Ich wünsche Ihnen, dass Sie gesund bleiben und mit Ihren Patientinnen und Patienten gemeinsam gut durch die Krise kommen.

\section{Mit freundlichen Grüßen}

Walter Struhal

\section{Korrespondenzadresse}

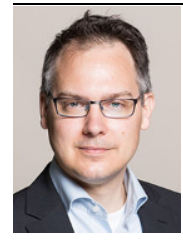

Prim. Assoc. Prof. PD Dr. Walter Struhal, FEAN

Abteilung für Neurologie,

Universitätsklinikum

Tulln, Karl Landsteiner

Privatuniversität für Gesundheitswissenschaften Alter Ziegelweg 10, 3430 Tulln, Österreich walter.struhal@tulln.lknoe.at

Interessenkonflikt. W. Struhal gibt an, dass kein Interessenkonflikt besteht.

\section{Literatur}

1. Varatharaj A et al (2020) Neurological and neuropsychiatric complications of COVID-19 in 153 patients: a UK-wide surveillance study. LancetPsychiatry. https://doi.org/10.1016/S22150366(20)30287-X

2. Grisold W, Struhal W, Grisold T (2019) Advocacy in neurology. Oxford University Press, Oxford

Hinweis des Verlags. Der Verlag bleibt in Hinblick auf geografische Zuordnungen und Gebietsbezeichnungen in veröffentlichten Karten und Institutsadressen neutral.

Hier steht eine Anzeige.

\section{Springer}

